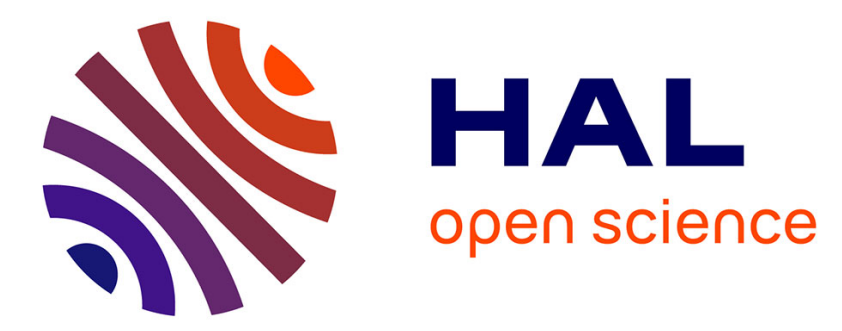

\title{
Le rôle des outils de coordination urbanisme-transports collectifs dans la fabrique politique urbaine
}

Caroline Gallez, Juliette Maulat, Olivier Roy-Baillargeon, Mariane Thébert

\section{To cite this version:}

Caroline Gallez, Juliette Maulat, Olivier Roy-Baillargeon, Mariane Thébert. Le rôle des outils de coordination urbanisme-transports collectifs dans la fabrique politique urbaine. Flux - Cahiers scientifiques internationaux Réseaux et territoires, 2015, 101-102, pp.5-15. 10.3917/flux.101.0005 . halshs01261266

\section{HAL Id: halshs-01261266 \\ https://shs.hal.science/halshs-01261266}

Submitted on 16 Mar 2018

HAL is a multi-disciplinary open access archive for the deposit and dissemination of scientific research documents, whether they are published or not. The documents may come from teaching and research institutions in France or abroad, or from public or private research centers.
L'archive ouverte pluridisciplinaire HAL, est destinée au dépôt et à la diffusion de documents scientifiques de niveau recherche, publiés ou non, émanant des établissements d'enseignement et de recherche français ou étrangers, des laboratoires publics ou privés. 
Introduction au numéro 101-102 de la revue Flux, 2015, pp. 5-15

\title{
Le rôle des outils de coordination urbanisme-transports collectifs dans la fabrique politique urbaine
}

\author{
Caroline Gallez, Juliette Maulat, Olivier Roy-Baillargeon et Mariane Thébert
}

\begin{abstract}
Dans les milieux professionnels comme dans la sphère de l'expertise, la nécessité de mieux coordonner l'urbanisme et les transports collectifs relève d'un postulat indiscutable à partir duquel se déclinent divers modèles, normes ou orientations de politique publique. Figure emblématique de la ville durable, la ville orientée vers les transports collectifs présenterait de multiples vertus : limiter la dépendance à l'automobile et ses effets négatifs en matière de consommation d'énergie et de pollution; lutter contre le mitage urbain en favorisant la structuration du développement urbain périphérique autour des pôles desservis en transport collectif; réguler les besoins de déplacement en encourageant l'émergence d'une urbanisation dense, mixte et favorable aux modes de déplacement collectifs et actifs. Au cours des vingt dernières années, de nombreux travaux ont été consacrés aux formes et effets potentiels d'un nouveau modèle d'urbanisation combinant la maîtrise de la croissance périurbaine et le développement d'axes de transport collectif. Ce modèle propose de dépasser celui de la ville compacte, généralement circonscrit aux zones centrales denses, pour lui substituer un schéma de développement multipolaire à l'échelle métropolitaine dans lequel le développement urbain se concentre autour des gares.
\end{abstract}

\section{La ville orientée vers les transports collectifs : émergence d'un nouveau modèle d'urbanisation}

Dans le domaine des transports, l'émergence de ce modèle d'urbanisme est associée à la remise en cause du principe «predict and provide» (prévoir et satisfaire les besoins de déplacements), qui a prévalu depuis les années 1950 et dont l'objectif était d'adapter l'offre d'infrastructures - principalement routières - à la croissance urbaine. Dans les années 1990, au-delà des préoccupations environnementales, le constat de l'inefficacité des stratégies d'augmentation de l'offre vis-à-vis de la réduction de la congestion routière, démontré par de nombreux travaux scientifiques, s'est peu à peu diffusé au sein de la sphère administrative (Goodwin et alii, 1991, Banister, 1994 ; Owens 1995). D'autres indices révèlent une remise en cause globale de cette politique de l'offre : la multiplication des critiques et des mobilisations contre les projets d'infrastructures, une préoccupation croissante concernant la pollution de l'air et la santé, la dénonciation des nuisances liées à la circulation automobile dans les zones urbaines et une critique de la dépendance des modes de vie à la mobilité.

Ce changement s'accompagne de la généralisation de la notion de mobilité durable (sustainable mobility), qui s'impose peu à peu dans les vocabulaires politique, technique, scientifique et citoyen (Bourdages, Champagne, 2012). Cette expression est utilisée pour la première fois dans un document officiel dans le Livre vert de la Commission européenne relatif à l'impact du transport sur l'environnement (CEC, 1992). Les auteurs du Livre vert soulignent que les efforts accomplis en matière de réduction de la pollution risquent d'être réduits à néant par l'augmentation globale de la mobilité liée à la croissance économique. Pour être atteints, les objectifs de mobilité durable exigent l'adoption de mesures additionnelles, telles que l'orientation des investissements vers le développement des modes de transports collectifs et la mise en œuvre d'une planification urbaine et régionale permettant de limiter le besoin de mobilité.

Aux États-Unis, la nécessité de promouvoir un mode de développement urbain radicalement opposé au modèle américain de la ville étalée est portée par les représentants de deux mouvements urbanistiques et architecturaux (New Urbanism et Smart Growth) qui préconisent un renouvellement des concepts et pratiques de l'urbanisme (Carlton, 2007). Le principe d'une urbanisation structurée autour des réseaux de transport collectif, inspiré des règles d'aménagement qui ont guidé le développement des villes européennes et américaines avant l'essor de l'automobile, a été formalisé par le concept de «Transit-Oriented Development » (TOD). Élaboré par l'architecteurbaniste américain Peter Calthorpe (1993), ce concept renvoie à l'aménagement de quartiers situés autour de stations de transport collectif respectant plusieurs qualités dont la densité, la mixité fonctionnelle ainsi qu'un 
environnement favorable aux déplacements à pied et à un usage combiné des transports publics, du vélo, de la marche et de la voiture.

À partir des années 2000, ce renouvellement des doctrines de l'urbanisme et de la planification des transports s'est accompagné d'un foisonnement de nouveaux outils d'action publique élaborés par les institutions étatiques, les gouvernements métropolitains ou les collectivités locales afin de favoriser la coordination urbanisme-transport. Leur visée est plus ou moins opérationnelle et leur portée juridique, variable. Le TOD a ainsi été traduit sous forme de normes et de recommandations dans des guides d'aménagement publiés par les agences métropolitaines des villes australiennes, canadiennes et états-uniennes puis diffusé par de multiples relais institutionnels aux échelles locale, nationale et internationale afin de faciliter sa mobilisation dans l'élaboration de plans directeurs ou d'opérations d'aménagement concrètes (Cervero et alii, 2004). En Suisse, dans le cadre de la nouvelle politique des agglomérations mise en place au début des années 2000, la contribution financière de la Confédération helvétique au financement de nouvelles infrastructures de transport est conditionnée à l'élaboration de projets d'agglomération favorisant une densification urbaine autour des stations. En France, les collectivités locales ont été à l'origine d'un instrument original, le contrat d'axe, qui formalise, dans un engagement réciproque des acteurs du transport et de l'aménagement, les contreparties territoriales (sous forme de densification de l'urbanisation, d'aménagement des pôles d'échange, etc.) d'une amélioration de la desserte en transport collectif. En Île-de-France, les contrats de développement territorial (CDT), inscrits dans la loi d'aménagement du Grand Paris, visent la signature d'accords entre la Préfecture d'Île-de-France et l'État sur la concentration d'une partie des logement neufs autour des gares du futur métro automatique. Certains outils visent une articulation sous l'angle financier. Suivant l'exemple pionnier du programme «Rail + Property » appliqué à Hong Kong dès les années 1980, des stratégies de financement des réseaux de transport ferroviaire à partir de la récupération des valeurs foncières sont mises en place dans plusieurs métropoles asiatiques (Singapour, Tokyo, Beijing, Shanghaï, etc.) à l'initiative de groupes privés intervenant à la fois dans les transports et la promotion immobilière (Cervero, Murakami, 2008).

Au cours des quinze dernières années, de nombreux travaux scientifiques ou rapports d'expertise ont été consacrés au suivi des expérimentations et à l'évaluation de l'efficacité de ces outils, notamment le TOD. Une part importante de ces travaux rend compte des expériences menées dans différentes villes étrangères et évalue leurs effets tout en cherchant à identifier les facteurs de succès ou de reproductibilité de ces expériences (Cervero et alii, 2004 ; Curtis, Renne, Bertolini, 2009). En France, le programme de recherche-action franco-allemand Bahn-Ville, associant des chercheurs et des acteurs de l'aménagement a également exploré les conditions favorables à l'avènement d'une «ville orientée vers le rail » (L'Hostis, 2009). D'autres travaux visent à évaluer de manière plus spécifique les impacts du TOD sur la part modale des transports collectifs, l'évolution du prix du foncier, ou encore les processus de gentrification ( $c f$. Kahn, 2007 ; Duncan, 2011).

Parmi ces recherches, rares sont celles qui s'intéressent à la dimension politique, c'est-à-dire aux modalités de l'action collective associées à la conception et à la mise en œuvre de ces outils de coordination. Cette action collective peut s'entendre à la fois sous la forme de démarches organisées, de politiques publiques ou de projets en vue d'atteindre des objectifs partagés, mais aussi de mouvements de résistance aux normes ou aux pouvoirs institués. L'hypothèse que nous formulons et qui a motivé l'appel à contributions à l'origine de ce numéro thématique est que cette dimension politique est fondamentale pour appréhender les enjeux, contraintes et conditions du changement dont ces outils sont éventuellement porteurs. Au-delà de la vision standardisée véhiculée par une doctrine ou un instrument d'action publique, c'est à travers les logiques et pratiques des acteurs publics et privés que se construit la relation entre l'urbanisme et les transports collectifs. La manière dont les outils de coordination contribuent à la fabrique des politiques urbaines doit être appréhendée à l'épreuve des situations locales.

Les auteurs de ce numéro thématique se sont emparés de cette problématique sous différents angles et à différentes échelles. Walter et Roy-Baillargeon analysent l'évolution de l'organisation institutionnelle et du cadre juridique liée à l'élaboration de deux générations successives de projets d'agglomération dans quatre agglomérations suisses. Douay et Roy-Baillargeon se penchent également sur les changements des institutions et des configurations d'acteurs associés à l'introduction des principes du TOD dans les deux dernières générations des documents de planification métropolitaine à Montréal. Delpirou, Doulet et Zhuo s'intéressent à la diffusion des enjeux d'articulation de l'urbanisme et des transports collectifs en Chine et illustrent les conditions de mise en œuvre de stratégies d'action coordonnées dans diverses métropoles chinoises. Enright analyse les représentations, discours et dispositifs concrets de la «métropole connectée et compétitive » du Grand Paris à travers la perspective de la théorie urbaine critique. En mobilisant le cadre théorique des instruments d'action publique, les contributions suivantes se focalisent sur la conception et la mise en œuvre des outils de coordination. Dushina, Paulhiac-Scherrer et 
Scherrer analysent le changement des modalités d'action collective associé à un projet de TOD dans la banlieue de Montréal. Maulat retire de l'étude croisée de plusieurs contrats d'axe ferroviaires une analyse des effets de ces expériences sur le changement de l'action publique en matière de transport ferroviaire et d'aménagement. Desrochers et Gauthier, pour leur part, interrogent la contribution des centres de gestion des déplacements (CGD) québécois à l'avènement d'une gestion des déplacements plus transversale et orientée vers l'usage des modes alternatifs à l'automobile. Les deux contributions finales offrent un retour d'expérience sur les outils de coordination de l'urbanisme et des transports collectifs par des acteurs publics participant à la diffusion des bonnes pratiques ou à leur expérimentation à l'échelle locale. Bentayou, Perrin et Richer proposent une mise en perspective des démarches de TOD et des contrats d'axe en s'appuyant sur différentes études de cas locales, dans les métropoles nord-américaines et dans plusieurs agglomérations françaises. Enfin, l'entretien avec Sophie Laurent et Juliana Ribeiro permet de découvrir les modalités originales de la démarche partenariale du " contrat aménagementtransport » expérimenté sur le projet d'extension de la ligne 11 du métro dans la petite couronne francilienne.

Les enseignements de ces différentes contributions sont abordés à travers le prisme de quatre entrées thématiques : la coordination de l'urbanisme et des transports collectifs comme question politique, les interactions entre outils de coordination et gouvernance métropolitaine, les changements opérés par ces outils dans la régulation de l'interface entre les réseaux et territoires et enfin, les limites de ces outils.

\section{La coordination de l'urbanisme et des transports collectifs : une question politique située dans le temps et dans l'espace}

La lecture des articles de ce numéro thématique vient conforter un constat déjà établi par des travaux antérieurs : loin de se résumer à ses aspects morphologiques ou techniques, la coordination de l'urbanisme et des transports collectifs est une question politique. Ses enjeux, ses mutations et sa traduction en actions concrètes ne peuvent donc être appréhendés en dehors des héritages et des contextes cognitifs et territoriaux dans lesquels elle se déploie, aux échelles tant nationale que locale.

Force est de constater, tout d'abord, la remarquable continuité dans le temps de la problématique de la coordination, véritable leitmotiv de la planification urbaine, qui s'oppose à l'évolution des objectifs parfois contradictoires qui lui ont été assignés au cours des dernières décennies. Si le mot d'ordre de la coordination s'entend principalement aujourd'hui en référence à la mobilité durable, il renvoyait dans les années 1950 à l'enjeu de l'adaptation des villes face à l'augmentation des circulations automobiles. Ce contraste invite dès lors à s'intéresser à l'évolution, sur le temps long, des cadres cognitifs ou des « référentiels » des politiques de transport et d'aménagement dans laquelle cette coordination s'insère (Gallez, Kaufmann, 2010). C'est cette perspective historique qu'adoptent Delpirou, Doulet et Zhuo (2015*) (1) lorsqu'ils rappellent qu'en Chine, l'émergence de l'enjeu de la coordination de l'urbanisme et des transports collectifs est liée à l'avènement d'un nouveau modèle d'urbanisation, dit modèle «intégrationniste», qui cherche à rompre avec un double héritage fonctionnaliste et «développementaliste». L'adoption de nouvelles mesures en matière de coordination urbanisme-transport en Chine peut difficilement se comprendre en dehors de l'ambition politique de ce modèle intégrationniste ; de la même manière, les contraintes à sa mise en œuvre doivent être appréhendées au regard de la persistance des héritages des modèles d'urbanisation précédents.

Au-delà du poids de ces héritages cognitifs, les enjeux et les formes de la coordination de l'urbanisme et des transports collectifs sont directement influencés par les éléments spatiaux, matériels, économiques, politiques et sociaux qui servent de cadre aux politiques locales (Kaufmann et alii, 2003 ; Nessi, Delpirou, 2009). Certains éléments sont susceptibles de constituer des facteurs d'irréversibilité, de contrainte ou, au contraire, d'opportunité pour les politiques publiques, soulignant dès lors une forme de dépendance au sentier des stratégies locales de coordination (Pflieger et alii, 2007). Dans les villes chinoises, en particulier, les transports collectifs peinent à s'insérer dans une structure urbaine à maille très large, où la monumentalité des blocs résidentiels, la hiérarchie des types de voirie, sont encore très marquées par «les logiques de stock et la valorisation extensive du foncier » (Delpirou, Doulet, Zhuo, 2015*). Plus largement et sans tomber dans une vision déterministe, l'inertie des modes de production de la ville, la lenteur de la transformation des infrastructures et du bâti ainsi que les contextes géographiques, constituent des contraintes pour les acteurs locaux et contribuent à une différenciation territoriale des stratégies de coordination de l'urbanisme et des transports collectifs

Cette différenciation, qui contraste avec la similitude des discours et doctrines de la ville orientée vers le rail, ressort tout particulièrement des études de cas présentées dans les différents articles de ce numéro thématique. Walter et 
Roy-Baillargeon $\left(2015^{*}\right)$ insistent sur la diversité de la prise en compte des enjeux de coordination dans les quatre agglomérations suisses qu'ils étudient, qui dépendent à la fois de contraintes physiques, du degré de continuité des politiques de transport ainsi que de traditions plus ou moins anciennes de planification territoriale et d'interventionnisme foncier. Au-delà de la diffusion d'orientations globales, la mise en cohérence des politiques d'urbanisme et de transports collectifs dépend avant tout des dysfonctionnements et des problèmes qui ont été énoncés localement (Gallez et alii, 2013). Ainsi, même si le concept de TOD est très largement repris dans le plan métropolitain d'aménagement et de développement (PMAD) du Grand Montréal, comme le montrent Douay et RoyBaillargeon $\left(2015^{*}\right)$, sa mise en œuvre sous forme d'opérations d'aménagement concrètes résulte, selon Dushina, Paulhiac-Scherrer et Scherrer (2015*), de négociations entre les acteurs, en fonction d'opportunités, d'enjeux et de contraintes locales. Bentayou, Perrin et Richer (2015*) constatent également la grande diversité des réalisations nord-américaines qui se réfèrent à ce modèle de développement, tout comme celle des expérimentations locales du contrat d'axe en France. Entre les acteurs du transport et de l'aménagement, l'accord initial autour de la coordination se limite généralement aux principes. Dans les faits, tant les problèmes à résoudre que les objectifs et les moyens de cette coordination ne sont ni donnés d'avance, ni limités aux aspects concrets ou techniques, certes cruciaux, des espaces fonciers disponibles ou des caractéristiques de l'infrastructure de transport : les enjeux touchent également au logement, au développement économique, à l'accessibilité aux ressources, à la qualité de vie, à l'attractivité des espaces urbains (Gallez, Thébert, 2013 ; Maulat, 2014).

Si l'écart entre l'intention planificatrice et sa mise en pratique donne lieu à diverses interprétations de la part des auteurs de ce numéro, tous s'accordent sur le constat de la diversité des enjeux associés par les acteurs locaux à la coordination. Douay et Roy-Baillargeon (2015*), à propos du PMAD, et Maulat (2015*) sur les usages locaux des contrats d'axe, montrent que cette diversité découle en partie des intérêts divergents des acteurs participant à ces démarches. Alors que les acteurs de l'aménagement s'emparent du TOD ou du contrat d'axe pour limiter l'éparpillement de l'urbanisation, favoriser la hiérarchisation urbaine autour de pôles secondaires ou encourager de manière sélective la densification autour des stations de transport collectif, les acteurs du transport visent avant tout l'amélioration des conditions de déplacement et l'augmentation de la fréquentation des transports collectifs. Delpirou, Doulet et Zhuo (2015*), à propos des villes chinoises, et Enright $\left(2015^{*}\right)$, sur le Grand Paris, insistent pour leur part sur le poids du développement économique et de la valorisation des espaces fonciers liés à l'aménagement autour des projets de transport. En Chine, l'enjeu de la valorisation foncière est particulièrement marqué lorsqu'il s'associe à une stratégie de récupération des plus-values afin de financer les réseaux, comme l'illustre le cas de la ligne 13 du métro de Beijing. Dans le contexte nord-américain de forte participation des acteurs privés à la définition des enjeux associés au TOD, Bentayou, Perrin et Richer (2015*) montrent que l'objectif d'attractivité commerciale de l'opération immobilière guide la transformation des abords des stations de transport collectif. Selon Enright (2015*), l'attractivité métropolitaine constitue l'enjeu central du Grand Paris. En s'appuyant sur la théorie urbaine critique, elle insiste sur la nature ambivalente de la « ville réticulaire » (networked city) et sur le rôle prépondérant des réseaux dans la pensée urbaine et dans la planification, en lien avec la valorisation positive de la mobilité. En améliorant la mobilité, le projet du Grand Paris servirait moins les intérêts des résidents de la région urbaine (ou d'une partie d'entre eux) que des objectifs de compétitivité métropolitaine, de revitalisation économique de la région Île-de-France, et de compétition à l'échelle internationale.

Au-delà de l'objectif normatif et spécialisé de la coordination entre transports collectifs et urbanisme, ces outils interviennent dans la régulation et dans les transactions institutionnelles entre secteurs et échelles d'action. Selon des modalités diverses, ils peuvent influencer les rapports de force entre acteurs du transport et de l'aménagement, ou entre les acteurs publics et privés. Les articles de ce numéro interrogent les interactions entre l'usage de ces outils, la réorganisation des institutions territoriales et les processus qui contribuent à la gouvernance métropolitaine.

\section{Coordination de l'urbanisme et des transports collectifs, institutions et gouvernance}

Depuis les années 1990, dans un contexte de transformation rapide des modes d'action collective liée à la globalisation économique, à l'européanisation et à la décentralisation des compétences de l'État, de nombreuses recherches ont été consacrées aux enjeux de régulation publique, de gouvernance et de gouvernabilité des réseaux et des territoires métropolitains. La gouvernance des grands réseaux techniques a notamment été appréhendée par la prise en compte du rôle des opérateurs, de la libéralisation et de la régulation socioéconomique des réseaux techniques et, plus généralement, de leur contribution à la fabrique urbaine, dans ses dimensions physique et politique (Gariépy, Marié, 1997 ; Coutard, 1999). 
L'une des questions récurrentes associées à la gouvernabilité métropolitaine porte sur l'organisation institutionnelle comme contrainte ou ressource de l'action collective urbaine. Plusieurs recherches ont appliqué cet angle d'analyse à la problématique de la coordination urbanisme-transport. À partir d'analyses comparatives sur des métropoles européennes, Sager (2006) a montré que la fragmentation géographique et technique des compétences, la segmentation des cultures professionnelles entre le transport et l'aménagement ainsi que le poids de la bureaucratie constituent généralement des freins à la mise en œuvre de stratégies d'action coordonnées. Delpirou, Doulet et Zhuo (2015*) formulent le même constat lorsqu'ils estiment qu'en Chine, au stade actuel de la diffusion de l'enjeu de la coordination de l'urbanisme et des transports collectifs, les impulsions mises en place à l'échelle nationale ne permettent pas de s'affranchir d'un «compartimentage administratif profondément inscrit dans les pratiques locales ». De leur côté, Douay et Roy-Baillargeon $\left(2015^{*}\right)$ soulignent que les divergences de vision des acteurs des différents paliers et secteurs d'action du Grand Montréal limitent la mise en place de stratégies de coordination des acteurs pour la constitution de quartiers TOD.

Le rôle de l'organisation institutionnelle comme levier du changement a toutefois été nuancé par d'autres travaux qui, sans remettre en cause son poids dans les modalités de l'action collective locale, ont montré que l'existence d'institutions disposant de compétences intégrées à l'échelle de l'agglomération ne constitue une condition ni suffisante ni nécessaire à la coordination (Gallez et alii, 2013). De ce fait, l'urgence serait moins de réfléchir à la création d'un nouveau «design institutionnel» favorable à la coordination qu'aux moyens de favoriser la coopération entre les institutions existantes. Legacy, Curtis et Sturup (2012) ont également souligné, dans le contexte australien, que l'intégration des autorités de l'urbanisme et du transport collectif ne conduit pas nécessairement à la mise en cohérence des politiques alors que la mise en réseau des parties prenantes couplée à une réglementation stricte apparaît plus efficace pour favoriser la coordination. Pour Dushina, Paulhiac-Scherrer et Scherrer $\left(2015^{*}\right)$, le cas du quartier TOD de Sainte-Thérèse montre que la fragmentation institutionnelle n'est pas un frein à la coordination et que le changement des pratiques d'aménagement peut s'opérer dans le cadre réglementaire existant, qui s'adapte par la suite au besoin. L'analyse par Walter et Roy-Baillargeon $\left(2015^{*}\right)$ des générations successives des projets de quatre agglomérations suisses vient appuyer ces résultats en mettant en évidence l'apprentissage collectif qui caractérise l'évolution des pratiques de la planification territoriale en Suisse depuis leur élaboration. Le renforcement des institutions régionales, lorsqu'il se produit, consiste en une institutionnalisation de pratiques de coordination antérieures : comme le soulignent les auteurs, en Suisse plus qu'ailleurs, en raison de l'autonomie communale et de l'attachement à la démocratie locale, la consolidation des prérogatives supracommunales reste en effet soumise à un fort contrôle démocratique.

Les outils de coordination urbanisme-transport telles que les centres de gestion des déplacements, des projets d'agglomération, du TOD ou des contrats d'axe, ont émergé face à la fragmentation sectorielle et géographique des compétences, La mise en œuvre de ces outils s'accompagne alors de la création de «scènes » d'interactions spécifiques entre acteurs. Des comités de pilotage des contrats d'axes aux tables régionales de concertation montréalaises, l'enjeu est de faire travailler ensemble les autorités organisatrices des transports, les opérateurs de transport, les autorités métropolitaines et les municipalités. Dushina, Paulhiac-Scherrer et Scherrer (2015*), Maulat (2015*), ainsi que Laurent et Ribeiro (2015*) évoquent la création de structures de gouvernance dédiées aux démarches partenariales ou contractuelles qui favorisent le décloisonnement des services techniques, les échanges entre les décideurs au-delà de leur seul domaine d'intervention et, parfois, le développement de nouvelles compétences. Desrochers et Gauthier $\left(2015^{*}\right)$ illustrent ainsi le rôle des CGD québécois dans la mise en contact et le montage de partenariats entre les acteurs participant à la mise en place des plans de gestion des déplacements d'entreprises : l'entreprise qui incite ses employés à recourir à des modes alternatifs à l'automobile, les autorités organisatrices de transport collectif qui agissent sur la tarification, les opérateurs de transport qui ont la possibilité de renforcer la qualité de desserte, les municipalités qui aménagent l'espace et développent les équipements publics adaptés ainsi que les usagers qui donnent leur avis sur ces services et aménagements. Walter et Roy-Baillargeon $\left(2015^{*}\right)$ insistent quant à eux sur le fait que les mécanismes particuliers de mise en œuvre des projets d'agglomération suisses jettent les bases de réformes institutionnelles transposant à l'échelle régionale des prérogatives et préoccupations auparavant confinées aux échelles communale ou cantonale.

Les articles attestent par ailleurs de différences importantes concernant les systèmes d'acteurs engagés dans ces dispositifs de coordination et, en particulier, la place accordée aux acteurs privés. Le regard croisé entre l'Amérique du Nord, l'Asie et l'Europe révèle des cultures de planification et de projet contrastées au regard de la place accordée aux acteurs privés. Dushina, Paulhiac-Scherrer et Scherrer $\left(2015^{*}\right)$ évoquent la place des promoteurs dans la négociation du projet de TOD de Sainte-Thérèse, tandis que l'approche transversale de Bentayou, Perrin et Richer 
(2015*) confirme qu'il s'agit là d'une caractéristique récurrente des opérations nord-américaines qualifiées de TOD. Ils soulignent également l'importance, dans le contexte nord-américain, des institutions « hybrides » (de statut privé ou associatif, et poursuivant des objectifs d'intérêt public ou communautaire) qui, en décalage avec la culture française de l'opposition (théorique) entre sphères publique et privée, contribuent à ouvrir le jeu des acteurs et intérêts représentés. Delpirou, Doulet et Zhuo $\left(2015^{*}\right)$ indiquent que la participation des opérateurs privés aux projets liant l'urbanisme et les transports collectifs est plutôt encouragée. En analysant la manière dont les acteurs concernés s'emparent de ces outils, les articles révèlent alors les rapports de force et tensions qui peuvent accompagner le déploiement de ces outils.

Les avantages supposés des outils de coordination de l'urbanisme et des transports collectifs dans la gouvernance des politiques urbaines fait alors l'objet de critiques. Pour Enright $\left(2015^{*}\right)$, le transport agit comme une institution matérielle qui modifie les relations de pouvoir au sein des espaces urbains et sur les territoires politiques locaux. Elle insiste sur la manière dont ce « régime de mobilité » (Böhm et alii, 2006) oriente les objectifs de la gouvernance métropolitaine. Se démarquant d'une vision étroite, instrumentale et fonctionnelle de la gouvernance et s'appuyant sur une vision foucaldienne des relations de pouvoir, l'auteure affirme que les contrats de développement territorial (CDT) autour des gares du Grand Paris Express sont un instrument au service d'une gouvernementalité urbaine en région Île-de-France, qui sert à (re)produire des idéaux, comportements et rationalités. On retrouve ainsi dans les dispositifs élaborés au nom de la coordination de l'urbanisme et des transports collectifs le risque d'instrumentalisation à propos de la diffusion des démarches de projet (Pinson, 1999) : l'approche de la gouvernance dont ils sont porteurs fait référence avant tout aux enjeux d'efficacité des politiques publiques - et plus particulièrement de renforcement de la capacité d'action en matière de gestion des grands réseaux techniques -, en occultant ceux du renouvellement des relations entre société civile et pouvoir politico-administratif.

\section{Les outils de coordination comme traceurs et vecteurs de changement}

Les outils de coordination de l'urbanisme et des transports collectifs sont-ils des traceurs ou des vecteurs de changement dans la manière de réguler les interfaces entre réseaux et territoires ? Sur ce point, les articles de ce numéro apportent des éclairages nuancés concernant les effets de ces outils sur les référentiels, les pratiques et le contenu des politiques.

Plusieurs auteurs montrent que la mobilisation et l'usage des outils de coordination contribuent à sensibiliser les acteurs aux enjeux de durabilité et de lutte contre l'éparpillement de l'urbanisation. Douay et Roy-Baillargeon $\left(2015^{*}\right)$ font l'hypothèse que la constitution d'aires TOD dans le PMAD du Grand Montréal permet à la fois de rendre plus visible et plus tangible la stratégie métropolitaine de densification tout en limitant sur des espaces restreints les éventuelles polémiques autour de cet objectif. Il semble en effet que cet outil ait permis d'appuyer l'acceptabilité par les acteurs municipaux du PMAD et du gel du périmètre d'urbanisation par le gouvernement. Desrochers et Gauthier $\left(2015^{*}\right)$ soulignent quant à eux que les processus d'élaboration des plans de gestion des déplacements, en associant institutions et entreprises, facilitent la diffusion des principes de la mobilité durable. Pour autant, cette sensibilisation est le plus souvent indirecte : c'est à travers les préoccupations des représentants des entreprises ou de leurs employés, et non par l'entremise d'un prosélytisme environnemental, que les acteurs des CGD québécois incitent à un changement des pratiques de déplacement. En d'autres termes, l'adhésion des acteurs aux enjeux de coordination de l'urbanisme et des transports collectifs dépend avant tout de la manière dont les outils de coordination s'adaptent aux problèmes qu'ils doivent résoudre localement. Maulat $\left(2015^{*}\right)$ montre que les contrats d'axe français favorisent l'adhésion des élus communaux aux enjeux de densification dès lors qu'ils rencontrent leurs préoccupations, en matière de diversification de l'offre de logement, par exemple. Ces démarches concourent également à une évolution des visions des acteurs du monde ferroviaire en révélant l'intérêt d'une prise en compte des évolutions territoriales autour du réseau ferré pour la définition d'une offre de transport adaptée aux besoins. Ce résultat rejoint les propos de Laurent et Ribeiro (2015*) selon lesquels l'expérience de contrat sur la ligne 11 du métro parisien a fait prendre conscience à l'autorité organisatrice des transports francilienne de l'intérêt qu'elle pouvait avoir à coupler le projet de transport à une réflexion sur le développement urbain autour de la ligne.

Certains articles de ce numéro apportent également des éléments de réponse concernant les effets de ces outils sur le changement des pratiques et les coopérations entre acteurs du transport et de l'aménagement. Pour Walter et RoyBaillargeon $\left(2015^{*}\right)$, l'évolution entre les première et deuxième générations de projets d'agglomération indique une forme d'apprentissage collectif impliquant un renforcement de la légitimité démocratique de ces dispositifs, une association plus étroite des communes et l'émergence d'un «nouveau régionalisme » à la suisse. Desrochers et Gauthier (2015*) montrent que la réalisation des plans de gestion des déplacements permet aux acteurs de 
confronter et d'ajuster leurs intérêts, en fonction de leurs besoins particuliers et dans le respect des limites de leurs ressources et compétences respectives. Selon Dushina, Paulhiac-Scherrer et Scherrer (2015*), l'expérience de TOD à Sainte-Thérèse a été le vecteur de coopérations nouvelles entre secteurs et échelles d'action, grâce à l'existence d'intérêts convergents et d'un portage politique fort du projet. Laurent et Ribeiro (2015*) insistent sur les effets d'acculturation des acteurs du transport et de l'aménagement au sein des groupes de travail thématiques mis en place à l'occasion de la démarche expérimentale de la ligne 11. Plus largement, les auteurs s'accordent autour de la capacité de ces démarches à faciliter la formulation des problèmes, révéler et confronter les intérêts contradictoires et, parfois, ouvrir la négociation politique. Si cette révélation suscite parfois des réticences, ainsi qu'en témoignent Laurent et Ribeiro $\left(2015^{*}\right)$ à propos des réserves foncières des communes, la démarche collective incite les acteurs politiques et techniques à anticiper les impacts de l'arrivée du métro sur le foncier, l'aménagement des stations et l'organisation du rabattement. Alors que les démarches des contrats aménagement-transport sont davantage orientées vers l'enrichissement de la «boite à outils » des acteurs locaux, les CDT, du fait de leur nature hybride entre norme et projet, contribuent à engager les débats sur des problèmes qui nécessitent un arbitrage politique, comme les objectifs de construction de logements ou la part de logement social (Gallez, Thébert, 2013).

Cependant, la manière dont ces négociations peuvent influencer le contenu des projets dépend de manière cruciale des rapports de force en présence et de l'ouverture des démarches à l'ensemble des acteurs concernés. Sans se prononcer sur les effets à long terme de ces outils, certains auteurs de ce numéro montrent ainsi que leur usage a, dans certains cas, permis d'influencer le contenu des projets vers une meilleure coordination de l'urbanisme et des transports collectifs. Dushina, Paulhiac-Scherrer et Scherrer $\left(2015^{*}\right)$ indiquent que la concrétisation d'un projet urbain présentant une densité supérieure autour de la gare de Sainte-Thérèse a été facilitée par des accords entre la municipalité et l'autorité organisatrice des transports collectifs ainsi que par l'intervention foncière de la municipalité. Maulat $\left(2015^{*}\right)$, de son côté, montre que les dispositifs de gouvernance, les outils de projet et le recours à une expertise dédiée déployés dans le cadre des contrats d'axe français est favorable à une amélioration de l'insertion territoriale de l'infrastructure ferroviaire, et, parfois, à l'augmentation des densités urbaines ou à la diversification des types de logements. Enright $\left(2015^{*}\right)$ estime au contraire que le contenu des projets des CDT est davantage orienté vers la prise en compte des intérêts économiques des investisseurs ou des entreprises ou des besoins d'une classe privilégiée qu'à ceux de la population résidente. De ce fait, les contenus de ces projets ne seraient durables ni d'un point de vue environnemental (du fait de l'extension de l'urbanisation et de la destruction d'espaces agricoles et d'écosystèmes), ni d'un point de vue social.

\section{Un changement, mais quel changement ?}

Finalement, les auteurs de ce numéro s'interrogent sur la nature et la portée des changements associés aux outils de coordination de l'urbanisme et des transports collectifs, et en soulignent notamment les limites. L'émergence de ces outils de coordination atteste une prise de conscience par les acteurs publics de la nécessité de faire évoluer les modes d'action collective dans un contexte de crise des finances publiques, d'aggravation de la pression environnementale et de renforcement de la compétition territoriale en matière de développement économique. Ces outils peuvent être instaurés par les acteurs nationaux, au nom du développement durable ou de la rationalisation des dépenses publiques (c'est le cas du TOD), mais ils peuvent également être «inventés » par les acteurs locaux, qui cherchent à dépasser les segmentations sectorielles par la mise en place de démarches partenariales ad hoc (c'est le cas des contrats d'axe et des contrats aménagement-transport en Île-de-France). Ce foisonnement de nouveaux outils d'action publique, d'initiative nationale ou locale témoigne bien d'un changement politique, répondant à la fois à une diversité d'enjeux globaux, de problèmes locaux et de besoins de coordination des acteurs dans un contexte d'hyper-fragmentation.

Les contributions de ce numéro mettent en évidence des formes de changement des pratiques et du contenu de l'action publique fortement dépendantes des contextes et systèmes d'acteurs locaux. La plupart des auteurs constatent des freins et inerties dans la mise en œuvre des outils liés aux organisations institutionnelles, résistances sectorielles, héritages territoriaux et questions de financement. Desrochers et Gauthier (2015*) attirent l'attention sur la difficulté des CGD québécois à faire appliquer ces principes en raison de leur rôle confiné à l'accompagnement et à la sensibilisation des entreprises et institutions, du manque de ressources consacrées par les employeurs à la concrétisation des plans élaborés pour eux ainsi que des capacités limitées d'intervention de leurs clients-partenaires. Maulat $(2015 *)$ rend compte des multiples contraintes de la coordination de l'urbanisme et du transport ferroviaire : multiplicité des temporalités, contraintes techniques, divergence des priorités, inerties cognitives, résistances institutionnelles, problèmes de financement, héritages fonciers territoriaux, etc. Elle souligne néanmoins que si les contrats d'axe ne réduisent pas la complexité liée à la fragmentation géographique et sectorielle des compétences, ils 
constituent un outil pragmatique de gestion et de régulation des décalages entre les échelles de définition et de gestion des problèmes. En s'appuyant sur le concept défini par Offner (1980), Dushina, Paulhiac-Scherrer et Scherrer $(2015 *)$ proposent de considérer le TOD comme un «opérateur local de congruence», soit un outil d'adaptation réciproque et de mise en correspondance résultante entre des logiques et des systèmes d'action différents. Dans cette perspective, le changement de politique s'opère grâce une démarche partenariale qui permet la convergence des intérêts différenciés des acteurs et la construction de l'interface entre réseaux et territoires en fonction des enjeux, des ressources et des contraintes locales.

Plusieurs auteurs s'interrogent sur l'efficacité des changements induits par ces démarches localisées, partielles ou incomplètes quant aux outils de mise en œuvre. Douay et Roy-Baillargeon $\left(2015^{*}\right)$ insistent sur le manque d'investissement dans l'offre de transport collectif et sur l'absence d'outils adéquats pour assurer la concrétisation des projets de TOD. La localisation des aires TOD actées dans le PMAD grand-montréalais paraît déjà porteuse de nombreux dysfonctionnements au regard des caractéristiques fonctionnelles du territoire et du réseau. Desrochers et Gauthier $\left(2015^{*}\right)$ soulignent que malgré les atouts des CGD quant à la diffusion des bonnes pratiques en matière de déplacement et à la facilitation de l'action collective, ils ne suffisent pas à concrétiser le renouvellement de la planification des transports, car ils gagneraient à être appuyés par d'autres outils réglementaires ou d'incitation économique.

Au-delà de la question de leur efficacité, les auteurs de ce numéro thématique remettent en cause l'idée selon laquelle ces outils s'accompagnent d'un réel changement de paradigme politique. Pour Douay et Roy-Baillargeon $\left(2015^{*}\right)$, le TOD constitue plus un «prétexte à la collaboration interscalaire en matière de transport et d'aménagement qu'un véritable instrument de mise en œuvre d'un urbanisme et d'une mobilité 'durables' ». Delpirou, Doulet et Zhuo (2015*) insistent sur la priorité accordée au développement économique dans le modèle d'urbanisation en Chine, avançant l'idée que «les impératifs d'urbanisation priment (encore) sur les enjeux d'urbanisme ». Maulat (2015*) souligne également que les contrats d'axe restent guidés par un paradigme de l'offre en matière de transport et de croissance en matière d'aménagement du territoire. Pour Enright $\left(2015^{*}\right)$, la question du changement s'entend à une échelle plus globale : en reprenant les termes de Garnier (2014), elle soutient l'idée selon laquelle les CDT relèvent d'un consensus centriste, d'une forme de gouvernance collaborative qui ne remet pas en cause les logiques d'action globales du capitalisme.

\section{Conclusion}

Les contributions de ce numéro illustrent les initiatives déployées par les gouvernements métropolitains et les acteurs locaux pour coordonner urbanisme et transport. Ces initiatives s'inscrivent dans un changement, sur le temps long, de l'approche des liens entre réseaux et territoires et correspondent à l'émergence d'un besoin de transversalité de l'action publique. Elles sont favorisées par un certain degré de souplesse du cadre législatif qui rend possible des arrangements et coopérations localisées entre acteurs au-delà des frontières institutionnelles. Ces expériences confirment alors que loin du mythe du périmètre pertinent et de la recherche permanente d'institutions à l'échelle des problèmes d'action publique, des actions collectives et des pratiques innovantes peuvent émerger «par le bas » pour dépasser les freins qui entravent la coordination urbanisme-transport.

Les effets des outils de coordination entre urbanisme et transports collectifs dépendent de leurs usages politiques et techniques et des contextes dans lesquels ils s'inscrivent. Les articles rassemblés ici révèlent que l'opérationnalité «aménagiste » des procédures, soit leur capacité à transformer les espaces, semble inversement proportionnelle à leur opérationnalité politique, c'est-à-dire à leur capacité à faire évoluer les modes d'action publique. De plus, le changement de politique opéré à travers ses outils ne s'accompagne pas d'un changement de paradigme mais se produit de manière progressive et incrémentale. Si les expérimentations présentées par les différents articles de ce numéro thématique attestent bien de ces évolutions partielles et parfois innovantes dans la manière de réguler l'interface entre les réseaux de transports collectifs et l'aménagement autour des gares, la question d'une régulation plus globale de ces expérimentations reste posée.

Ce numéro ouvre dès lors plusieurs pistes de recherches. Il invite, tout d'abord, à poursuivre l'analyse de ces outils d'action publique pour comprendre, sur le temps long, leur éventuelle contribution à un changement des pratiques. Dans la perspective de décloisonner la recherche sur les transports et l'aménagement, les résultats de ce numéro gagneraient également à être croisés avec d'autres travaux sur les interactions réseaux-territoires (par exemple sur les processus de requalification urbaine des infrastructures routières ou sur les interactions ville-port) mais aussi avec des recherches portant sur d'autres types de réseaux techniques. Par ailleurs, tout en mettant l'accent sur la dimension politique de la coordination entre urbanisme et transports collectifs, ce numéro n'aborde pas la question 
du rôle des citoyens. La question de la place des citoyens dans ces actions visant un modèle de métropole arrimée aux réseaux de transport collectifs mais également de leurs résistances et de leurs éventuelles contributions à la construction d'alternatives constituent sans aucun doute des pistes de recherche intéressantes. Enfin, l'analyse des projets ciblés d'articulation entre transports collectifs et urbanisme gagnerait à être prolongée par une réflexion sur le rôle de régulation des acteurs métropolitains ou régionaux dans le maintien de solidarités financières, sociales et spatiales, afin d'éviter le risque que se mette en place une nouvelle géographie prioritaire, au détriment des espaces qui se trouvent à l'écart des réseaux de transports collectifs.

Caroline Gallez Caroline Gallez est chercheuse à l'IFSTTAR, au Laboratoire Ville Mobilité Transport. Ses travaux portent sur l'analyse des politiques locales de déplacement, en lien avec les politiques d'aménagement et sur les politiques intercommunales. Elle a co-dirigé avec Vincent Kaufmann un ouvrage sur les mythes et pratiques de la coordination urbanisme-transport dans quatre agglomérations suisses et françaises. Sa thèse d'habilitation, soutenue en 2015 porte sur "La mobilité quotidienne en politique, des manières de voir et d'agir". caroline.gallez@ifsttar.fr

Juliette Maulat est docteure en aménagement de l'espace et urbanisme de l'Université Paris 1 et membre de l'UMR Géographie-Cités. Ses recherches concernent principalement la relation transport-aménagement, l'action publique locale et le monde ferroviaire. Sa thèse, soutenue en décembre 2014, s'intéresse aux référentiels, aux pratiques d'action et au contenu des politiques ferroviaires régionales et d'aménagement locales mises en cuvre dans les territoires métropolitains, au prisme des injonctions à la coordination urbanisme-transport. juliettemaulat@gmail.com

Olivier Roy-Baillargeon achève un doctorat en aménagement à l'Université de Montréal sous la direction du professeur émérite Michel Gariépy. Ses recherches portent sur la gouvernance de la planification métropolitaine, la coordination du transport et de l'aménagement et le TOD. Ses publications récentes traitent de l'importation, l'appropriation et l'instrumentalisation d'outils d'aménagement et d'urbanisme étrangers à des fins de construction politique par les décideurs et planificateurs des régions métropolitaines canadiennes. olivier.roybaillargeon@gmail.com

Mariane Thébert

Note

(1) Les références accompagnées d'un astérisque renvoient aux articles de ce numéro.

\section{Bibliographie}

Banister D., 1994, Reducing the Need to Travel through Planning, Town Planning Review, Vol. 65, № 4, p. 349354.

Bentayou G., Perrin E., Richer C., 2015, Contrat d'axe et Transit-Oriented Development : quel renouvellement de l'action publique en matière de mobilité et d'aménagement ?, Flux 2015/3-4 (n 101-102), p. ??-??.

Böhm S., Jones C., Land C., Paterson M. (eds.), 2006, Against Automobility, Malden: Wiley-Blackwell.

Bourdages J., Champagne É., 2012, Penser la mobilité durable au-delà de la planification traditionnelle du transport, VertigO - la revue en ligne des sciences de l'environnement, Hors-série 11 | mai 2012. DOI : 10.4000/vertigo. 11713

Calthorpe P., 1993. The next American metropolis : ecology, community, and the American dream, Princeton: Princeton Architectural Press.

Carlton I., 2007, Histories of Transit-Oriented Development : Perspectives on the Development of the TOD Concept. Real Estate and Transit, Urban and Social Movements, Concept Protagonist, Berkeley: IURD. 
CEC - Commission of the European Communities, 1992, Green Paper on the Impact of Transport on the Environment : A Community Strategy for 'sustainable mobility', COM (92) 46 final, 20 February 1992. [EU Commission - COM Document]

Cervero R., Murakami J., 2008, Rail+Property Development : a model of sustainable transit finance and urbanism, Working Paper, UC Berkeley Center for Future Urban Transport.

Cervero R., Murphy S., Ferrell C., Goguts N., Tsai Y.-H., Arrington G.B., Boroski J., Smith-Heimer J., Golem R., Peninger P., Nakajima E., Chui E., Dunphy R., Myers M., McKay S., Witenstein N., 2004, TransitOriented Development in the United States : Experiences, Challenges, and Prospects. Transit Cooperative Research Program (TCRP) Report 102, Washington: Transportation Research Board.

Coutard O., 1999, The Governance of Large Technical Systems, New York: Routledge.

Curtis C., Renne J. L., Bertolini L., 2009, Transit Oriented Development: Making it Happen, London: Ashgate.

Delpirou A., Doulet J.-F., Zhuo J., 2015, Coordonner urbanisme et transports collectifs : un référentiel à l'épreuve de la ville «made in China », Flux 2015/3-4 (n $101-102)$, p. ??-??.

Desrochers C., Gauthier M., 2015, Les centres de gestion des déplacements au Québec : un instrument de renouvellement de la planification des transports ?, Flux 2015/3-4 (n¹01-102), p. ??-??.

Douay N., Roy-Baillargeon O., 2015, Le Transit-Oriented Development (TOD), vecteur ou mirage des transformations de la planification et de la gouvernance métropolitaines du Grand Montréal ?, Flux, 2015/3-4 (n 101-102), p. ??-??.

Duncan, M., 2011, The impact of transit-oriented development on housing prices in San Diego, CA, Urban Studies, vol. 48, p. 101-127.

Dushina A., Paulhiac-Scherrer F., Scherrer F., 2015, Le TOD comme instrument territorial de la coordination entre urbanisme et transport : le cas de Sainte-Thérèse dans la région métropolitaine de Montréal Flux 2015/3-4 $\left(\mathrm{n}^{\circ} 101-102\right)$, p. ??-??.

Enright T., 2015, Transportation and the Coordination of the Competitive Parisian Metropolis, Flux 2015/3-4 $\left(n^{\circ} 101-102\right)$, p. ??-??.

Gallez C., Kaufmann V. (sous la direction de), 2010, Mythes et pratiques de la cohérence urbanisme-transport : regards croisés sur quatre agglomérations suisses et françaises, Rapports de recherche de l'INRETS, $\mathrm{N}^{\mathrm{o}} 281$, Mayenne : Jouve.

Gallez C., Kaufmann V., Maksim H.-N., Thébert M., Guerrinha C., 2013, Coordonner transport et urbanisme: Visions et pratiques locales en Suisse et en France, Revue d'économie régionale et urbaine, № 2, p. 317 337.

Gallez C., Thébert M., 2013, Les contrats de développement territorial (CDT) du Grand Paris : une négociation multi-échelles de l'articulation urbanisme-transport ? Le cas des CDT Cœur Descartes et Boucles de la Marne, Rapport final de contrat pour le MEEDDE, décembre 2013.

Gariépy M., Marié M. (sous la direction de), 1997, Ces réseaux qui nous gouvernent, Paris : L'Harmattan.

Garnier J-P., 2014, Le 'Grand Paris' : une urbanisation sans urbanité. Prémonitions lefebvriennes sur un avenir métropolitain, [On line] (Consulted on 31 August 2015) Available at: http://terrainsdeluttes.ouvaton.org/?p=2818?

Goodwin P. B., Hallett S., Kenny F., Stokes G., 1991, Transport : the new realism, Oxford: University of Oxford.

Kahn M. E., 2007, Gentrification trends in new transit-oriented communities: Evidence from 14 cities that expanded and built rail transit systems, Real Estate Economics, vol. 35, p. 155-182.

Kaufmann V., Sager F., Ferrari Y., Joye D., 2003, Coordonner transports et urbanisme, Lausanne : Presses polytechniques et universitaires romandes. 
Laurent S., Ribeiro J., 2015, Une démarche pilote portée par la Région Île-de-France : Charte et contrat aménagement-transport autour du prolongement de la ligne 11 du métro, Entretien réalisé par Caroline Gallez et Juliette Maulat, Flux 2015/3-4 (nº101-102), p. ??-??.

Legacy C., Curtis C., Sturup S., 2012, Is there a good governance model for the delivery of contemporary transport policy and practice? An examination of Melbourne and Perth, Transport Policy, Vol. 19, No 1, p. 8-16.

L'Hostis A. (sous la direction de), 2009, Concevoir la ville à partir des gares, Rapport final du projet Bahn-Ville.2, Predit-Deufrako.

Maulat J., 2015, Contractualiser pour coordonner urbanisme et transport ? Regards croisés sur quatre expériences de contrats d'axes ferroviaires, Flux 2015/3-4 (n¹01-102), p. ??-??.

Maulat J., 2014, Coordonner urbanisme et transport ferroviaire régional : le modèle à l'épreuve des pratiques. Etude croisée des métropoles de Toulouse et Nantes, Thèse de géographie et aménagement de l’Université de Paris 1 - Panthéon Sorbonne.

Nessi H., Delpirou A., 2009, Les politiques de « développement urbain durable » face aux héritages territoriaux. Regards romains sur la coordination transport/urbanisme, Flux 2009/1 (nº75), p. 69-79.

Offner J.-M., 1980, L'évaluation des investissements de transport : effets ou congruence, Colloque du GRECO CNRS Etudes de suivi et processus de décision, novembre.

Owens S., 1995, From "predict and provide" to "predict and prevent?" : Pricing and planning in transport policy, Transport Policy, Vol. 2, $\mathrm{N}^{\circ}$ 1, p. 43-49.

Pinson G., 1999, Projets urbains et construction des agglomérations : échelles fonctionnelles et politiques, Annales de la recherche urbaine, $\mathrm{N}^{\circ} 82$, p. 130-139.

Pflieger G., Kaufmann V., Jemelin C., Pattaroni L., 2007, Entre reproduction, innovation et contingence : infléchir une trajectoire urbaine. L'exemple des politiques locales de transports et d'urbanisme, Revue suisse de sociologie, Vol. 33, $\mathrm{N}^{\mathrm{o}}$ 3, p. 369-386.

Sager F., 2006, Policy coordination in the European metropolis : A meta-analysis, West European Politics, Vol. 29, $\mathrm{N}^{\circ} 3$, p. 433-460.

Walter S., Roy-Baillargeon O., 2015, La coordination du transport et de l'aménagement à l'heure des projets d'agglomération suisses : la régionalisation de la planification à Berne, Genève, Lausanne et Zurich, Flux 2015/3-4 (n¹01-102), p. ??-??. 